\title{
Discrete cortical regions associated with the musical beauty of major and minor chords
}

\author{
Miho Suzuki, Nobuyuki OKamura, Yousuke Kawachi, and Manabu Tashiro \\ Tohoku University, Sendai, Japan \\ Hiroshi Arao \\ Hiroshima International University, Hiroshima, Japan \\ TAKAYUKI Hoshishiba \\ Nippon Bunri University, Oita, Japan \\ AND \\ Jiro Gyoba ANd KAZUHIKo YANAI \\ Tohoku University, Sendai, Japan
}

\begin{abstract}
Previous research has demonstrated that the degree of aesthetic pleasure a person experiences correlates with the activation of reward functions in the brain. However, it is unclear whether different affective qualities and the perceptions of beauty that they evoke correspond to specific areas of brain activation. Major and minor musical keys induce two types of affective qualities — bright/happy and dark/sad — that both evoke aesthetic pleasure. In the present study, we used positron emission tomography to demonstrate that the two musical keys (major and minor) activate distinct brain areas. Minor consonant chords perceived as beautiful strongly activated the right striatum, which has been assumed to play an important role in reward and emotion processing, whereas major consonant chords perceived as beautiful induced significant activity in the left middle temporal gyrus, which is believed to be related to coherent and orderly information processing. These results suggest that major and minor keys, both of which are perceived as beautiful, are processed differently in the brain.
\end{abstract}

Neural correlates of aesthetic pleasure have been the subject of recent studies (Blood \& Zatorre, 2001; Blood, Zatorre, Bermudez, \& Evans, 1999; Brown, Martinez, \& Parsons, 2004; Jacobsen, Schubotz, Höfel, \& von Cramon, 2006; Kawabata \& Zeki, 2004; O’Doherty et al., 2003). Across the sensory modalities (e.g., music, visual art, an attractive face), brain activity correlated with perceived pleasure has been observed in several distinct areas, including the paralimbic and neocortical regions (Blood \& Zatorre, 2001; Blood et al., 1999; Brown et al., 2004; Kawabata \& Zeki, 2004; Menon \& Levitin, 2005; O’Doherty et al., 2003). Highly pleasurable objects increased and decreased brain activity in regions responsible for motivation, emotion, and arousal, including the ventral striatum, midbrain, amygdala, orbitofrontal cortex, and ventral medial prefrontal cortex. These results indicate that aesthetic pleasure activates the brain circuitry that is involved in emotion and reward. However, it is unclear whether different affective qualities and the perceptions of beauty that they evoke correspond to specific areas of brain activation.

Affective qualities refer to the various emotions and concepts represented by art that deeply touches our hearts.
Some types of art conceptualize negative affective feelings such as sadness, heart-wrenching sorrow, or fear, whereas other types represent the positive aspects of human life, such as happiness, love, and hope. In music, minor and major keys - the main musical components - are closely related to whether negative or positive affective feelings are being expressed in musical pieces.

In music, a key is related to the subset of pitches selected in a given musical segment; minor keys are associated with a sad sound, whereas major keys are associated with a happy sound (see Crowder, 1984, for a review). Much of Western music can be divided into major and minor keys, both of which evoke considerable aesthetic pleasure even though their affective qualities are quite different (Crowder, 1984; Gagnon \& Peretz, 2003; Hevner, 1935). However, little is known about the brain activity responsible for the experience of these musical keys.

In this study, we investigated the neural correlates of the perception of the beauty of major and minor keys by using $\left[{ }^{15} \mathrm{O}\right] \mathrm{H}_{2} \mathrm{O}$ positron emission tomography (PET). We used musical chords as stimuli in order to clearly define a major or minor key through the use of consonance (perceived as beautiful) and dissonance (perceived

M.Suzuki, suzukim@sal.tohoku.ac.jp 
as ugly), thus enabling the simple manipulation of the music's aesthetic quality. We used chord sequences in normal or variant progression patterns. We obtained PET scans of 13 male participants as they listened to four types of music-beautiful, major-key consonant (B-majC) chords; beautiful, minor-key consonant (B-minC) chords; ugly, major-key dissonant (U-majD) chords; and ugly, minor-key dissonant (U-minD) chords. Subtraction analyses were used to examine the participants' regional cerebral blood flow (rCBF) as they listened to each of the four types. In addition, the participants rated the beauty of the music on a scale of 1 (most beautiful) to 7 (most ugly). We also performed regression analyses of the subjective rating scores and the $\mathrm{rCBF}$ to investigate the relationships between them.

We conducted this study with the expectation that comparing the rCBF changes elicited by the beautiful consonant chords with those elicited by the ugly dissonant chords would reveal activation of the reward-related neural system, since such chords produce a pleasant or an unpleasant affective feeling. The most important aim of this study was to compare the rCBF changes elicited by listening to $\mathrm{B}-$ majC chords with those elicited by listening to B-minC chords. Such a comparison should elucidate the specific brain areas-beyond the general reward-related system - responsible for the appreciation of the major and minor musical keys.

\section{METHOD}

\section{Participants}

The participants comprised 13 healthy male students (20-26 years old) from Tohoku University-10 right-handed, 2 left-handed, and 1 ambidextrous. All of the participants had normal hearing capacity and no history of neurological or psychiatric disease. They had an average of 3.3 years of musical experience (including piano lessons), and 1 participant self-reported that he possessed perfect pitch. The study protocol was approved by the Clinical Research and Ethics Committee of Tohoku University. All of the participants provided written informed consent to participate in the study.

\section{Stimuli}

We composed a total of 96 chord sequences - 48 major-key consonant and dissonant chords and 48 minor-key consonant and dissonant chords. The dissonant sequences were created by raising the top tones of all of the chords in each sequence by a semitone. The pitch range extended from E3 to A5. In each sequence, we presented eight chords in succession at the rate of one chord every $1.5 \mathrm{sec}$, for a total duration of $12 \mathrm{sec}$. Each chord consisted of four tones played simultaneously on a computer that simulated the sound of a real piano with natural tone decay. For each of the major- and minor-chord sequences, the normal and variant progression patterns were specified. We duplicated each progression pattern, and each duplicate pattern included different inversion patterns to constitute the eight-chord sequence.

We conducted an initial screening with 139 listeners to select the major and minor subsets of sequences that scored highest in beauty and ugliness ratings. This screening yielded a subset of 32 sequences that comprised 8 major consonant, 8 major dissonant, 8 minor consonant, and 8 minor dissonant sequences.

We conducted an additional pilot study to investigate the affective qualities of these 32 chord sequences. Another group of 45 participants rated the 32 sequences on three 7-point scales ( $\mathrm{sad}$ to happy, dark to bright, depressive to hopeful) that were selected on the basis of previous research that investigated the affective qualities of musical keys. Since the ratings for the three scales had high correlation coefficients (ranging from .95 to .98 ), we averaged all of the participants' $(N=45)$ scores for the three scales and conducted a two-way ANOVA on the data, with major versus minor and consonance versus dissonance as within-subjects factors. The results revealed significant main effects and interactions. Major consonant chords received the highest scores, which indicated very happy, bright, and hopeful feelings. In contrast, the minor chords in both the consonant and the dissonant versions received low scores, with no significant differences between them. Although the rating scores for the major dissonant chords were higher than those for the minor dissonant, there was a strong significant difference between the scores for the major consonant chords and those for the major dissonant chords.

We conducted a second screening with the 13 PET experiment participants in order to select each participant's five beautiful major, five beautiful minor, five ugly major, and five ugly minor chord sequences from the 32 subsets of consonant and dissonant sequences. None of the participants selected consonant chords as ugly or dissonant chords as beautiful. We assigned the selected sequences to the major consonant, major dissonant, minor consonant, and minor dissonant conditions for each participant in the PET experiment. Under each condition, the sequence perceived as the most beautiful or ugliest was played first, followed by the other four sequences played in an order determined by the proximity of their key to that of the initially played sequence. Finally, the initial sequence (most beautiful or ugliest) was replayed. We played these sequences with an intersequence interval of $3 \mathrm{sec}$, resulting in a total listening duration of $90 \mathrm{sec}$ under each condition. We started playing the music before starting the scans so that the participants would have begun experiencing emotions when the scan started.

\section{Image Acquisition and Procedure}

We obtained a 3-D PET using a SET2400W scanner (Shimadzu, Kyoto) in accordance with previously described methods (Okamura et al., 2000). We scanned the participants for $70 \mathrm{sec}$ after an intravenous administration of approximately $5 \mathrm{mCi}(185 \mathrm{MBq})$ of $\left[{ }^{15} \mathrm{O}\right] \mathrm{H}_{2} \mathrm{O}$ through an antecubital vein. A total of 10 scans was conducted; the first and last were done while the participants rested. During the remaining eight scans, the participants lay on their backs and were instructed to close their eyes and listen as the chord sequences corresponding to each of the four conditions were presented in a counterbalanced order. After each scan, we asked the participants to rate the beauty of the chord sequence on a 7-point scale (from $1=$ most ugly to $7=$ most beautiful).

\section{Statistical Analysis of PET Images}

We used SPM2 software for image realignment, normalization, and smoothing, and to create statistical maps of significant $\mathrm{rCBF}$ changes (Friston et al., 1994). Using nonlinear transformation, all of the CBF images were stereotactically normalized into the standard space of Talairach and Tournoux (1988). The normalized images were smoothed using a $12 \times 12 \times 12 \mathrm{~mm}$ Gaussian filter. The rCBF values, adjusted by the analysis of covariance, were expressed as $\mathrm{ml} \cdot \mathrm{dl}^{-1} \cdot \mathrm{min}^{-1}$, and were scaled to a mean of 50 . The $t$ statistics were computed for each voxel in order to draw a comparison among the four experimental conditions and the resting condition. To analyze these comparisons, the threshold for significant voxels was set at $p<.001$, uncorrected for multiple comparisons.

In this analysis, we hypothesized that the brain areas engaged in music listening would be the auditory cortices and the limbic and paralimbic regions, since a significant activation of these regions has been reported in previous PET and fMRI studies on music-listening tasks. At the cluster level, the threshold was set at a corrected $p<.05$ for all subtraction analyses in order to reduce accidental findings due to Type I errors. Pearson's simple correlation methods were used to assess the correlations of the voxel value and subjective rating scores; the threshold of significance was set at $p<.05$. 


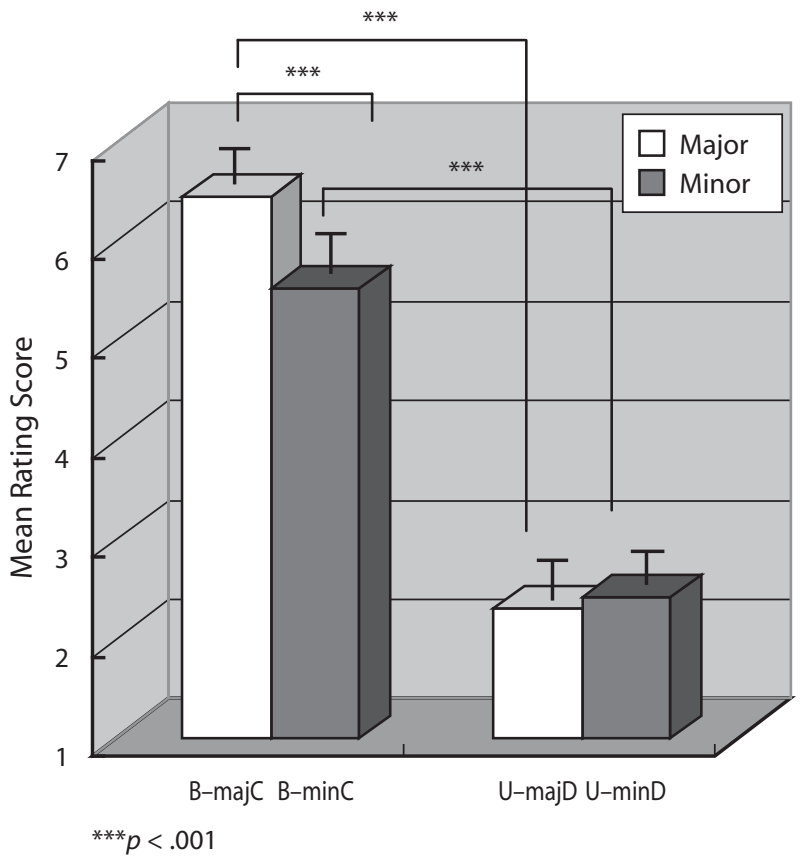

Figure 1. Mean rating scores of musical chord stimuli in each of the four conditions, based on the behavioral results obtained after each PET scan. Error bars represent the standard errors of the means for the relative conditions. $\mathrm{B}-\mathrm{majC}$, beautiful, majorkey consonant condition; $B-$ minC, beautiful, minor-key consonant condition; U-majD, ugly, major-key dissonant condition; U-minD, ugly, minor-key dissonant condition.

\section{RESULTS}

\section{Behavioral Results}

In the preliminary experiment, each participant selected his five beautiful and five ugly chords from among the 32 consonant and dissonant chords. To confirm that the participants experienced the beauty or ugliness of the chords as they listened to them during the PET scan, a two-way ANOVA (beautiful consonance vs. ugly dissonance $X$ major vs. minor) was performed for the subjective rating scores. The results revealed that the average rating score for the beautiful consonant chords was significantly higher than that for the ugly dissonant chords, regardless of the key (major or minor) $(p<.001$; Figure 1$)$. In addi- tion, the major chords generally received a higher rating than did the minor chords $(p<.001)$. The average rating score for the beautiful major consonant chords was higher than that for the beautiful minor consonant chords $(p<$ .001 ), whereas no significant difference was found between the mean ratings for the ugly major and minor dissonant chords. These results indicate that during the PET scans, the participants judged the beauty of the chords in a reliable and appropriate manner, as expected from the preliminary experimental manipulations.

\section{Imaging Results}

Differences between the music-listening and rest conditions. Initially, the rCBF images under the five conditions (B-majC, U-majD, B-minC, U-minD, and resting) were compared statistically to confirm the brain regions involved in the task of listening to musical chords. In comparison with the resting state, several regions of the temporal and frontal areas were found to be significantly activated during the four listening conditions, including the bilateral middle frontal gyrus and bilateral superior temporal gyrus $(30,40,-2, z=5.50, p<.001 ;-40,42$, $-2, z=4.35, p<.001 ;-46,2,-16, z=4.20, p<.001$; and $10,24,50, z=3.84, p<.001$, respectively).

Differences between beautiful music and ugly music. We conducted subtraction analyses to identify the brain areas that responded specifically to beautiful consonant music and those that responded specifically to ugly dissonant music. The coordinates and $z$ scores of the subtraction analyses are given in Table $1(p<.001$, uncorrected). Listening to beautiful consonant chords induced significant activities in the dorsomedial midbrain $(-10$, $-28,-14 ; z=3.97)$, whereas listening to ugly dissonant chords evoked significant activations of the right inferior parietal lobule (IPL) $(57,-32,53 ; z=3.56)$, left insula $(-46,-15,3 ; z=3.30)$, and left frontal subgyral $(-26$, 20,$26 ; z=3.30$ ) (Table 1). Moreover, the rCBF of these brain regions was significantly correlated with the subjective rating scores. The rCBF of the dorsomedial midbrain activated by the beautiful consonant chords, compared with that activated by the ugly dissonant ones, was positively correlated with the subjective rating scores $(p<.05)$. On the other hand, comparing the activations caused by the ugly dissonant chords with those caused by the beautiful consonant ones yielded significantly negative correlations

Table 1

Coordinates and $z$ Scores for the Brain Regions in Subtraction Analyses in Three Conditions: Beautiful Consonance Versus Ugly Dissonance, Ugly Dissonance Versus Beautiful Consonance, and Minor Key Versus Major Key

\begin{tabular}{llrrrr}
\hline \multicolumn{1}{c}{ Condition and Region } & & \multicolumn{3}{c}{ Talairach Coordinates } \\
\cline { 5 - 6 } & Hemisphere & $z$ Score & $x$ & $y$ & $z$ \\
\hline $\begin{array}{l}\text { Beautiful consonance vs. ugly dissonance } \\
\quad \text { Dorsomedial midbrain }\end{array}$ & Left & 3.97 & -10 & -28 & -14 \\
$\begin{array}{l}\text { Ugly dissonance vs. beautiful consonance } \\
\text { Inferior parietal lobule }\end{array}$ & Right & 3.56 & 57 & -32 & 53 \\
$\quad$ Insula & Left & 3.30 & -46 & -15 & 3 \\
$\quad$ Frontal subgyral & Left & 3.30 & -26 & 20 & 16 \\
$\quad$ Minor key vs. major key & & & & & \\
$\quad$ Parahippocampus & Left & 3.45 & -24 & -35 & -3 \\
\hline
\end{tabular}


between the rating scores and $\mathrm{rCBF}$ in the IPL/left insula $(p<.05)$.

Differences between major-chord music and minorchord music. We also conducted subtraction analyses to investigate the specific regions corresponding to major and minor musical chords. The coordinates and $z$ scores of the analyses are given in Table 1 ( $p<.001$, uncorrected). Subtraction of the signal evoked by minor chords from that elicited by major chords showed no significant activation; however, the inverse subtraction showed activation of the left parahippocampal gyrus $(-24,-35,-3 ; z=3.45)$. The activated areas revealed by subtraction analyses under the major- and minor-chord conditions included the brain regions activated by both the dissonant and the consonant chords. To create the dissonant chords, we raised the top tones of the original chords by a semitone; as a result, the original chord may have been unclear. Our pilot data support this conclusion. The subjective rating scores for the affective qualities of beautiful major consonant chords differed considerably from those for the affective qualities for minor chords; however, we did not find much difference between the scores for the ugly major and minor dissonant chords (see Method). Therefore, in our analysis of the representation of major and minor keys, we focused on the areas that were activated by listening to the $\mathrm{B}-$ majC chords and those activated by listening to the $\mathrm{B}-$ minC chords.

Differences between $B-$ majC music and $B-$ minC music. A subtraction analysis comparing the effects induced by the B-majC chords with those induced by the U-majD ones revealed that $\mathrm{rCBF}$ increased in the left middle temporal gyrus (MTG) $(-61,-1,-18 ; z=3.77)$ and the right fusiform gyrus $(61,-15,-30 ; z=3.33)(p<.001$, uncorrected; Table 2) in the $\mathrm{B}-\mathrm{majC}$ condition. A comparison between the $\mathrm{B}-\mathrm{majC}$ and $\mathrm{B}-\mathrm{minC}$ conditions also revealed significant $\mathrm{rCBF}$ in the left MTG $(-61,3,-20 ; z=3.46$; see Table 3 and Figure 2).

In contrast, significantly higher $\mathrm{rCBF}$ appeared in the left dorsomedial midbrain $(-10,-30,-13 ; z=3.92)$, left rectal gyrus $(-6,18,-28 ; z=3.78)$, right cerebellar tonsil $(14,-60,-32 ; z=3.60)$, right inferior frontal lobule $(32,31,2 ; z=3.54)$, left cingulate gyrus $(-4,-14$, $32 ; z=3.40)$, left anterior cingulate gyrus $(-8,33,-2$; $z=3.35)$, and right corpus striatum $(32,-9,10 ; z=3.34)$ (Table 2) during presentation of the $\mathrm{B}-$ minC chords than during presentation of the $\mathrm{U}-\mathrm{minD}$ chords. The right corpus striatum $(30,-15,11 ; z=4.39)$ and culmen $(-34$,

Table 3

Coordinates and $z$ Scores of Brain Regions: A Direct Comparison Between the Beautiful, Major-Key Consonant (B-majC) and the Beautiful, Minor-Key Consonant (B-minC) Conditions

Talairach

Coordinates

\begin{tabular}{|c|c|c|c|c|c|}
\hline \multirow[b]{2}{*}{ Condition and Region } & \multirow[b]{2}{*}{ Hemisphere } & \multirow[b]{2}{*}{$z$ Score } & \\
\hline & & & $x$ & $y$ & $z$ \\
\hline B-majC vs. B-minC & & & & & \\
\hline Middle temporal gyrus & Left & 3.46 & -61 & 3 & -20 \\
\hline B-minC vs. B-majC & & & & & \\
\hline Corpus striatum & Right & 4.39 & 30 & -15 & 11 \\
\hline Culmen & Left & 3.43 & -34 & -44 & -21 \\
\hline
\end{tabular}

Table 2

Coordinates and $z$ Scores for Brain Regions: A Direct Comparison Between the Beautiful, Major-Key Consonant (B-majC) Condition Versus the Ugly, Major-Key Dissonant (U-majD) Condition and the Beautiful, Minor-Key Consonant (B-minC) Condition Versus the Ugly, Minor-Key Dissonant (U-minD) Condition

\begin{tabular}{|c|c|c|c|c|c|}
\hline \multirow[b]{2}{*}{ Condition and Region } & \multirow[b]{2}{*}{ Hemisphere } & \multirow[b]{2}{*}{$z$ Score } & \multicolumn{3}{|c|}{$\begin{array}{c}\text { Talairach } \\
\text { Coordinates }\end{array}$} \\
\hline & & & $x$ & $y$ & $z$ \\
\hline \multicolumn{6}{|l|}{ B-majC vs. U-majD } \\
\hline Middle temporal gyrus & Left & 3.77 & -61 & -1 & 18 \\
\hline Fusiform gyrus & Right & 3.33 & 61 & -15 & -30 \\
\hline \multicolumn{6}{|l|}{ B-minC vs. U-minD } \\
\hline Dorsomedial midbrain & Left & 3.92 & -10 & -30 & -13 \\
\hline Rectal gyrus & Left & 3.78 & -6 & 18 & -28 \\
\hline Cerebellar tonsil & Right & 3.60 & 14 & -60 & -32 \\
\hline Inferior frontal gyrus & Right & 3.54 & 32 & 31 & 2 \\
\hline Cingulate gyrus & Left & 3.40 & -4 & -14 & 32 \\
\hline Anterior cingulate gyrus & Left & 3.35 & -8 & 33 & -2 \\
\hline Corpus striatum & Right & 3.34 & 32 & -9 & 10 \\
\hline
\end{tabular}

$-44,-21 ; z=3.43$ ) also displayed stronger significant activations during the $\mathrm{B}-$ minC condition than during the B-majC condition (see Table 3 and Figure 2).

To formally test the relationships between cortical activities and subjective beauty, we performed regression analyses that compared the participants' subjective rating scores with the $\mathrm{rCBF}$ they experienced while listening to musical chords. For the B-majC and U-majD conditions, we found significant positive correlations between the subjective ratings and cortical activities in the left MTG $(\beta=.315, p<.05)$ and the right fusiform gyrus $(\beta=$ $.383, p<.005)$. On the other hand, for the $\mathrm{B}-\operatorname{minC}$ and U-minD conditions, there were positive correlations between the subjective scores and the cortical activities in the left dorsomedial midbrain $(\beta=.468, p<.0001)$, left rectal gyrus $(\beta=.445, p<.001)$, right inferior frontal lobule $(\beta=.369, p<.007)$, left cingulate gyrus $(\beta=$ $.388, p<.005)$, and left anterior cingulate gyrus $(\beta=$ $.387, p<.005)$.

\section{DISCUSSION}

This study has revealed the cortical regions that are responsible for the affective processing involved in listening to musical chords. We found that beautiful consonant chords, regardless of their key, strongly activated the dorsomedial midbrain region, including the substantia nigra. This result is indeed consistent with results from previous research investigating musical pleasantness (Blood \& Zatorre, 2001; Blood et al., 1999; Menon \& Levitin, 2005), which suggested that the reward system is related to the experience of musical pleasure. The dopamine neurons located in the substantia nigra pars compacta and the ventral tegmental area project mainly to the striatum, ventral striatum, and frontal cortex, which are reported to construct a reward circuit (Schultz, 1999). Although it is difficult to identify the substantia nigra pars compacta as the activation foci because of the limitation of the PET spatial resolution, our results suggest the possibility that 

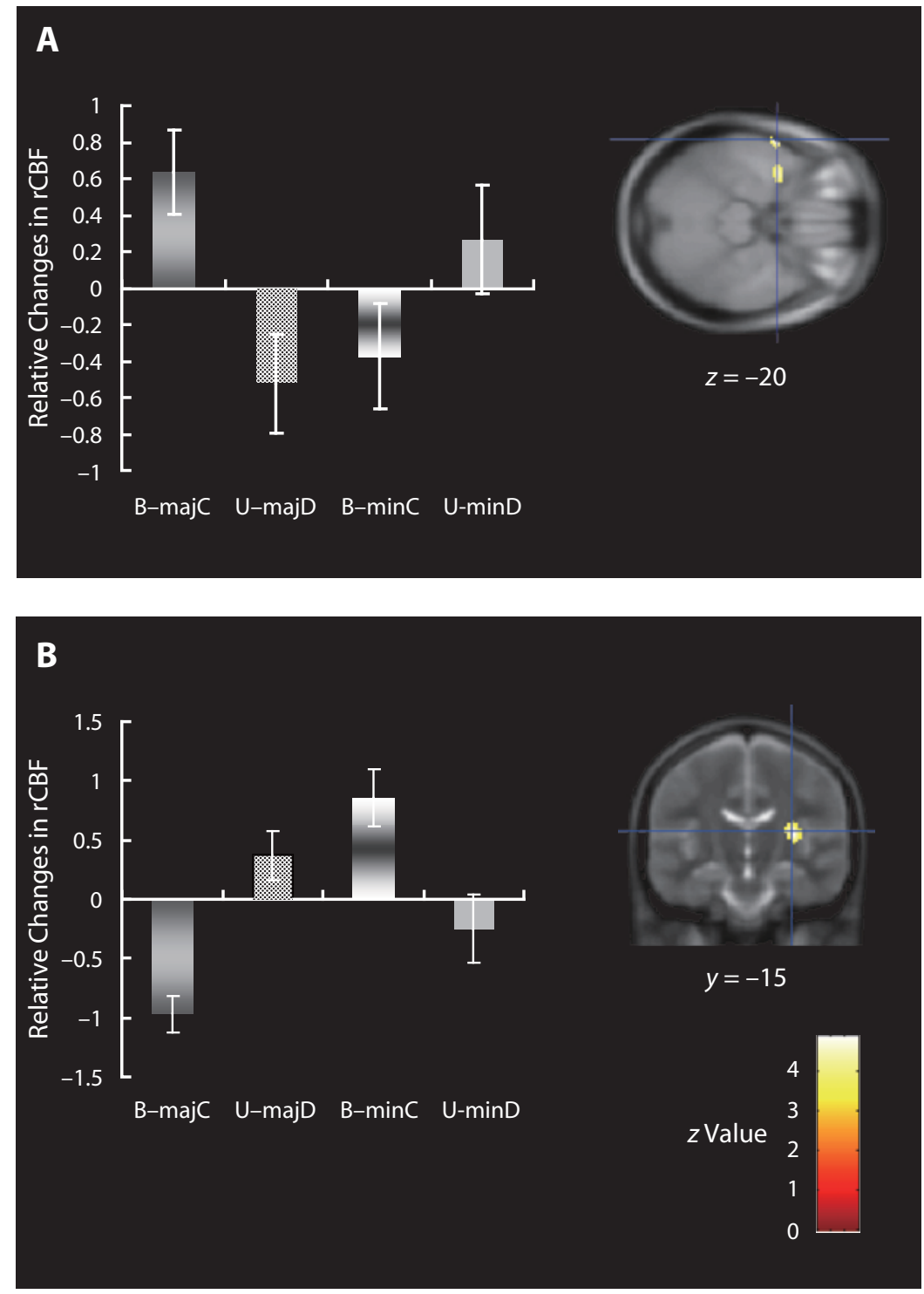

Figure 2. Neuroanatomical regions demonstrating significant increases in $\mathbf{r C B F}$ upon the subtraction of the $B$-minC condition images from the $B-$ majC ones $(A)$ and vice versa $(B)$. In the $B$-majC condition, as compared with the $B$-minC condition, the $\mathrm{rCBF}$ increased in the left middle temporal gyrus (axel section; $z=-20 \mathrm{~mm}$ ), whereas in the $\mathrm{B}-$-minC condition, as compared with the $\mathrm{B}-$-majC condition, the $\mathrm{rCBF}$ increased in the right corpus striatum (coronal section; $y=-15 \mathrm{~mm}$ ). Error bars represent the standard errors of the means for the relative conditions. $\mathrm{CBBF}$, regional cerebral blood flow; see Figure 1 for explanations of the other acronyms.

the dopaminergic reward system plays an important role in processing the pleasure that is evoked by listening to musical chords.

More specifically, beautiful minor consonant chords, in comparison with ugly minor dissonant ones, strongly activated several distinct spatial regions associated with reward and emotion, including the dorsomedial midbrain, right inferior frontal lobule, and anterior cingulate gyrus, whereas minor dissonant chords did not strongly activate these areas. In contrast, major consonant chords did not induce significant activations of these areas, despite the fact that the subjective beauty scores for the major consonant chords were significantly higher than those for the minor consonant ones. These results suggest that the beauty of major musical keys and that of minor keys are represented in different areas of the brain, and the activations of the reward nerve system do not always have to correspond to the intensity of the subjective feeling of beauty.

A comparison of the activations evoked by the beautiful minor-key music and those evoked by the beautiful majorkey music revealed that the striatum is an important site for the processing of the former, whereas the MTG is an important site for the processing of the latter. The striatum, which is projected by the dopaminergic neurons of the 
midbrain, is one of the most important regions involved in operating the reward system. Therefore, pleasure evoked by music in the minor key is akin to reward and may stir up strong emotions in humans. Traditionally, in Japan, the type of beauty evoked by minor-key music - for example, mono no aware (the pathos or sadness of things, i.e., lacrimae rerum) and wabi-sabi (austere simplicity tinged with elegant melancholy) (Sano, 1995) - is more dominant in art than is the type of beauty evoked by major-key music. It is possible that the strong activation of the reward circuit for minor-key beauty in the present study was due to cultural bias. Further investigation is required in order to examine the interaction between the neural networks and cultural differences in perceptions of beauty.

Consonant major keys also activate distinct areas of the brain, specifically the left MTG, which is reported to mediate word processing - in particular, orthographic word processing and morphologic information processing (Gernsbacher \& Kaschak, 2003; Sakai, Hashimoto, \& Homae, 2001; Vigneau, Jobard, Mazoyer, \& TzourioMazoyer, 2005). The left MTG appears to be related to coherent and orderly information processing. In the theory of harmonics, a major triad appears to exhibit more natural and fundamental characteristics of the physical nature of sound than does a minor triad (Crowder, 1984). The major triad occurs naturally in the harmonic series as the fourth, fifth, and sixth partials, whereas complex analyses are necessary to identify the corresponding minor triad. In light of these considerations, it seems possible that listening to beautiful major-key music mediates coherent information processing.

Our results suggest that the beauty of the major and minor musical keys is represented in different brain areas, which correspond to their different affective qualitiesthat is, happy/sad, bright/dark, and hopeful/depressive. Many things in life, such as visual art, music, faces, nature, and mathematics, induce feelings of beauty; such beautiful things activate not only the reward nerve system (Blood \& Zatorre, 2001; Blood et al., 1999; Brown et al., 2004; Kawabata \& Zeki, 2004; O’Doherty et al., 2003) but also other areas that mediate various affective qualities. Previous researchers who have examined the neurological basis of experiences of beauty have not focused sufficiently on the diverse manifestations of such experiences. Our findings primarily demonstrate that the different affective qualities of major and minor musical keys, both of which are perceived within the realm of beauty, are represented in distinct brain regions. This differential representation might underlie, in part, the depth and complexity of aesthetic pleasure evoked by art.

\section{AUTHOR NOTE}

This study was supported by a Japan Society for the Promotion of Science research fellowship for young scientists (184848) to the first author and by a Ministry of Education, Culture, Sports, Science, and Technology grant-in-aid for scientific research (18650063) to the sev- enth author. Correspondence concerning this article should be addressed to M. Suzuki, Department of Pharmacology, Tohoku University School of Medicine, 2-1, Seiryo, Aoba-ku, Sendai, Miyagi 980-8575, Japan (e-mail: suzukim@sal.tohoku.ac.jp).

Note-This article was accepted by the previous editorial team, when John Jonides was Editor.

\section{REFERENCES}

BLood, A. J., \& ZATorre, R. J. (2001). Intensely pleasurable responses to music correlate with activity in brain regions implicated in reward and emotion. Proceedings of the National Academy of Sciences, 98, 11818-11823.

Blood, A. J., Zatorre, R. J., Bermudez, P., \& Evans, A. C. (1999). Emotional responses to pleasant and unpleasant music correlate with activity in paralimbic brain regions. Nature Neuroscience, $\mathbf{2}$, 382-387.

Brown, S., Martinez, J. M., \& Parsons, L. M. (2004). Passive music listening spontaneously engages limbic and paralimbic systems. NeuroReport, 15, 2033-2037.

Crowder, R. G. (1984). Perception of the major/minor distinction: I. Historical and theoretical foundations. Psychomusicology, 4, 3-12.

Friston, K. J., Holmes, A. P., Worsley, K. J., Poline, J.-P., Frith, C. D., \& FrackowiaK, R. S. J. (1994). Statistical parametric maps in functional imaging: A general linear approach. Human Brain Mapping, 2, 189-210.

Gagnon, L., \& Peretz, I. (2003). Mode and tempo relative contributions to "happy-sad" judgments in equitone melodies. Cognition \& Emotion, 17, 25-40.

Gernsbacher, M. A., \& KaschaK, M. P. (2003). Neuroimaging studies of language production and comprehension. Annual Review of Psychology, 54, 91-114.

Hevner, K. (1935). The affective character of the major and minor modes in music. American Journal of Psychology, 47, 103-118.

Jacobsen, T., Schubotz, R. I., Höfel, L., \& von Cramon, D. Y. (2006). Brain correlates of aesthetic judgment of beauty. NeuroImage, 29, 276-285.

KaWabata, H., \& ZeKi, S. (2004). Neural correlates of beauty. Journal of Neurophysiology, 91, 1699-1705.

Menon, V., \& Levitin, D. J. (2005). The rewards of music listening: Response and physiological connectivity of the mesolimbic system. NeuroImage, 28, 175-184.

O’Doherty, J., Winston, J., Critchley, H., Perrett, D., Burt, D. M., \& Dolan, R. J. (2003). Beauty in a smile: The role of medial orbitofrontal cortex in facial attractiveness. Neuropsychologia, 41, 147-155.

Okamura, N., Yanai, K., Higuchi, M., Sakai, J., Iwata, R., Ido, T., ET AL. (2000). Functional neuroimaging of cognition impaired by a classical antihistamine, $d$-chlorpheniramine. British Journal of Pharmacology, 129, 115-123.

SAKaI, K. L., Hashimoto, R., \& Homae, F. (2001). Sentence processing in the cerebral cortex. Neuroscience Research, 39, 1-10.

SANO, K. (1995). Japanese mentality and behavior-based on the indigenous Japanese culture. Acta Neurochirurgica, 132, 192-195.

Schultz, W. (1999). The reward signal of midbrain dopamine neurons. News in Physiological Sciences, 14, 249-255.

TAlairach, J., \& Tournoux, P. (1988). Co-planar stereotaxic atlas of the human brain: 3-dimensional proportional system. An approach to cerebral imaging (M. Rayport, Trans.). Stuttgart: Thieme.

Vigneau, M., Jobard, G., Mazoyer, B., \& Tzourio-Mazoyer, N. (2005). Word and non-word reading: What role for the Visual Word Form Area? NeuroImage, 27, 694-705.

(Manuscript received January 17, 2007; revision accepted for publication August 14, 2007.) 